\title{
ARTICLE
}

\section{The Current Status and Reinforcement Plan for Radiation Emergency Medicine in Korea}

\author{
Hyun Ki Kim ${ }^{*}$, Youngmin Lee ${ }^{2}$ and Jai Ki Lee ${ }^{2}$ \\ ${ }^{1}$ Korea Institute of Radiological and Medical Sciences, Seoul, Korea \\ ${ }^{2}$ Hanyang University, Haengdang-dong, Seongdong-gu, Seoul, Korea
}

\begin{abstract}
Korea operating twenty nuclear power plants was ranked $6^{\text {th }}$ in nuclear power generation in the world. The potential risk for radiological emergency also increases along with the growing use of radiation and radioisotopes and a constant expansion of the nuclear industry in neighboring countries, Japan and China. This paper is intended for finding ways to strengthen medical planning and response preparedness from achievements in radiation emergency medicine over the years. 'Integrated Medical Preparedness System' for a radiological disaster is proposed as a practical way to enhance mobilization of existing human and material resources in the health care. It is based on the collaborative response among the related medical institutes : radiation emergency medical institutes around the Korean Institute of Radiological and Medical Sciences, emergency medical centers around the National Emergency Medical Center and other hospitals available.
\end{abstract}

KEYWORDS: radiological disaster, radiation emergency, medical preparedness, radiological emergency planning

\section{I . Introduction}

Korea operating twenty nuclear power plants was ranked $6^{\text {th }}$ in nuclear power generation in the world. As use of radiation and radioisotopes in the fields of industry, medical care, research and education increases, the number of institutes using them comes to about 2,800. In addition, Northeast Asia is expected to be a high density area of nuclear power plant. With the use of nuclear and radiation, the risk of terror which is becoming a global problem lately also causes an increase in the incidence possibility of radiological disaster.

After Chernobyl nuclear accident, it is emphasized that the need of medical care system to radiation emergencies that can cause mass casualties. Each of the East Asian countries like Japan, China and Taiwan has been constructed its own system using various medical resources to provide advanced radiation emergency medicine.

In Korea, since the 'Law on Protection of Nuclear Facilities and Countermeasure for Radioactive Preparedness ${ }^{1)}$ was enacted in 2003, nationwide radiation emergency medical system including the Korean Institute of Radiological and Medical Sciences (KIRAMS) has been established for the medical response in radiological disaster due to nuclear accident, radioactive terror, etc.

This paper suggests the ways to establish practical medical preparedness system by considering the status of the Korean radiation emergency medicine based on the 'Method for the development of emergency response preparedness for nuclear or radiological accidents' that the IAEA recommends ${ }^{2)}$.

*Corresponding author, E-Mail: hkkim@kirams.re.kr

(C) Atomic Energy Society of Japan

\section{Principles of Radiological Emergency Planning}

In order to be effectively implemented, emergency medical response should be planned and organized in accordance with particular infrastructure and functional elements. Being the part of overall emergency preparedness and response, medical response should meet the same approach to requirements as it's given in the IAEA TECDOC-953 for all response organization. The infrastructure elements must be in place to ensure the objectives of response can be performed in the event of an emergency. They consist of authority, organization, coordination, plans and procedures, logistical support, training and drill, etc., as shown in Figure 1.

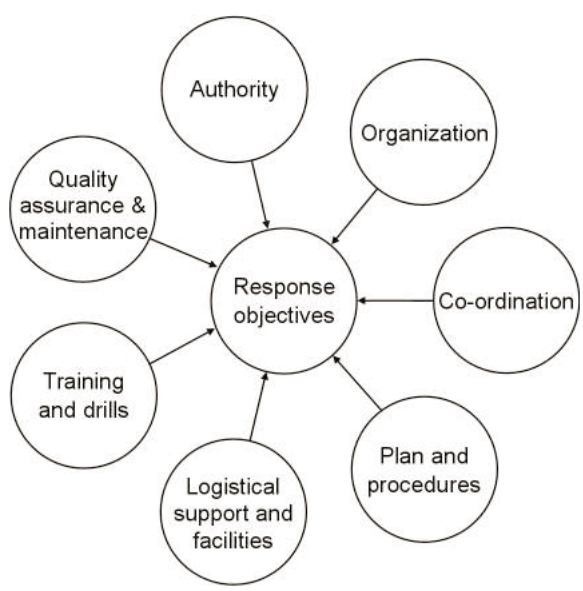

Fig. 1 Infrastructure elements to achieve response objectives

\section{Status of Radiation Emergency Medicine}

The KIRAMS of the Ministry of Education, Science and Technology (MEST) and 18 hospitals have been operated 
to provide emergency medical services in a radiological disaster. As shown in Figure 2, 5 small- and medium-sized hospitals near the nuclear power plant were designated as primary radiation emergency medical institute and 13 local main hospitals were designated as secondary radiation emergency medical institute. The KIRAMS manages the nationwide network for radiation emergency medicine and is responsible for all of the radiation emergency medical care during an actual emergency.

Although efforts for establishing the system was started without any of the basis for radiation emergency medicine, it is definite there must be viable achievements as follows.

- Securing a local stronghold for radiation medicine in a radiological accident

- Consolidating a foundation for radiation medicine by training the medical personnel and supporting equipments

- Collaborating with international agencies and other countries such as WHO, IAEA, Japan, USA, etc.

Meanwhile the role of the Ministry for Health, Welfare and Family Affairs (MIHWAF) which actually implements medical relief for the public has been relatively reduced because the MEST is in charge of the radiological accident. It leads to some problems limiting medical resources that might be mobilized in accidents and reducing the level to respond radiological emergency by itself. It means infrastructure elements such as organization, co-ordination and logistical support are not fully considered within medical organizations to fulfill response objectives.

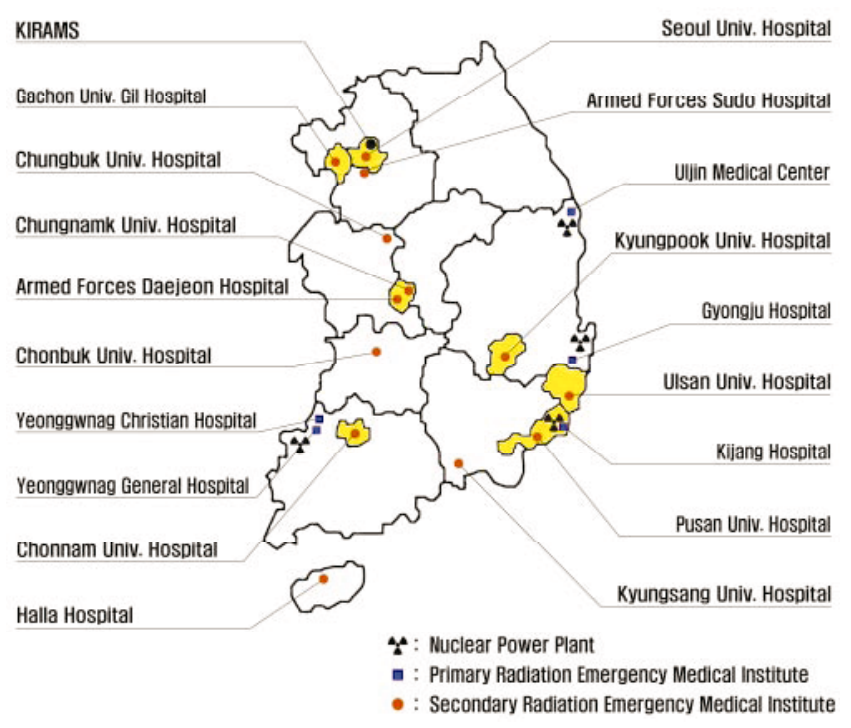

Fig. 2 Radiation emergency medical institutes

\section{Available Medical Resources in Radiological disaster}

\section{Emergency Medical System}

Current emergency medical system in Korea is built with the 'Emergency Medical Law'. The National Emergency Medical Center (NEMC), 16 regional emergency centers and 98 local emergency centers were selected and have been operated. The law obliges those centers to support emergency medicine in a disaster as a one of their duties.

\section{Resources for Specialized Treatment}

The change in the blood due to bone marrow damage is the typical pathology from overexposed patients. The antiseptic room and drugs to recover hematogenous functions are required for the patient who has severe bone marrow damage. The major general hospitals in the country are running the bone marrow transplantation center that has about 10 20 wards in different center.

\section{Emergency Medical System of Nuclear Power Plant Operator}

Korea Hydro and Nuclear Power Co. has the Radiation Health Research Institute (RHRI) that is specialized organization to study the health effect of radiation and has been concluding an agreement with 10 hospitals near the nuclear power plant to provide nuclear workers with medical services in a small accident. This system does not establish direct relations with national radiation emergency medical system but it is a one of the resources available in a radiological disaster.

\section{Integrated Medical Preparedness System}

It can not be expected in practice to cover medical needs with only national radiation emergency medical system in the radioactive disaster. The current medical infrastructure could be consolidated through cooperation with the emergency medicine that reserves considerable medical resources already and emergency medical system that the operator of a nuclear power plant has established. It is rather normal for radiation emergency medicine in association with emergency medicine to respond a radiological disaster as mentioned in the Emergency Medical Law.

The problem is lack of mutual understanding about their respective roles and the need for cooperation because radiation emergency medicine and emergency medicine are conceptually divided in two due to different authorities. In the national crisis, radiological disaster, it is sure that medical activities are going to be done without any distinction between them. Therefore, the roles and relationship between two systems taking into account their own specific goals and responsibility during the planning process should be documented in the response plans and procedures based on agreements with the NEMC which plays a central nervous part in emergency medical system.

Figure 3 shows the emergency medical system suggested with the concept of integrated medical response in a radioactive disaster. In the radiological disaster, the National Radiological Emergency Management Committee is set up in the MEST and the National Emergency Medical 
Committee is set up in the MIHWAF. The National Emergency Medical Committee will be also responsible for radiation emergency medicine if necessary as well as emergency for a traumatic injury or other conventional injury. Emergency medicine is conducted by the emergency medical centers and radiation emergency medical institutes with an assistance of the NEMC of the MIHWAF. The Radiological Emergency Medical Service Center performs a double function, medical treatment for the evacuated casualties and technical support for radiation emergency medicine including dosimetry and request for international assistance from Radiation Emergency Medical Preparedness and Assistance Network of WHO.

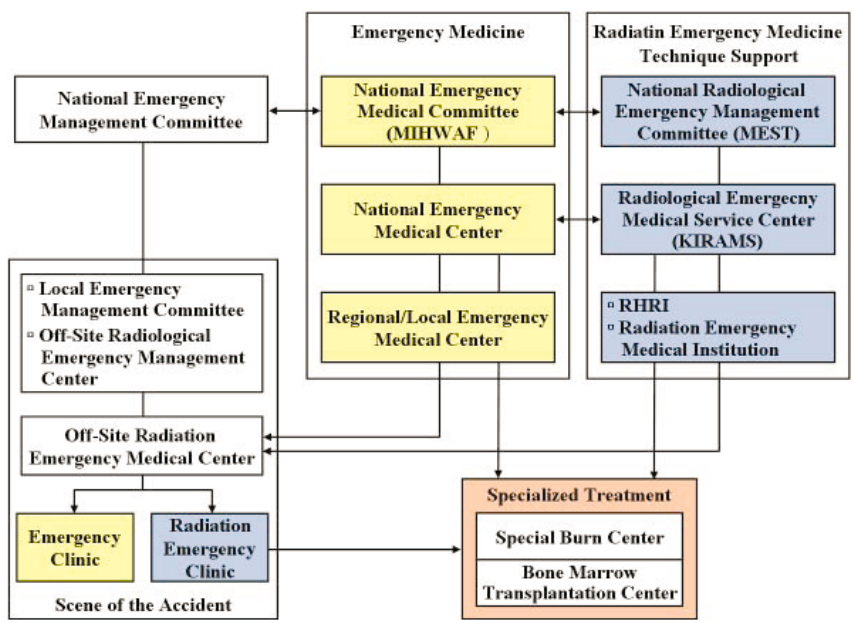

Fig. 3 Integrated medical preparedness system in a radiological disaster

\section{Conclusions}

Currently, the medical relief in a radiological disaster such as nuclear accident and radiological terror is completely dependent on the national radiation emergency medical system around the KIRAMS. However it is inevitable for emergency medical system to be activated in a radiological disaster not to predict the size of casualties. 'Integrated Medical Preparedness System' is suggested as a practical way to enhance mobilization of existing human and material resources in the health care in an emergency and to better prepare against a radiological disaster. It is based on the collaborative response among the related medical institutes : radiation emergency medical institutes around the KIRAMS, emergency medical centers around the NEMC which is playing an essential role in the national emergency medicine and other hospitals available. This system of a new concept improves the medical response capability in a radiological disaster in both quality and quantity, which is also an effective way to reduce the social and economical cost on responding a rare event.

\section{References}

1) Government of the Republic of Korea, "Law on Protection of Nuclear Facilities and Countermeasure for Radioactive Preparedness", Atomic Energy Act No. 7428 (2005).

2) IAEA, "Method for the Development of Emergency Response Preparedness for Nuclear or Radiological Accidents", International Atomic Energy Agency, IAEA-TECDOC-953 (1997) 